\title{
The issues of designing of construction features of the internal combustion cam engine
}

On the pages of the article described cam-piston mechanism developed as an alternative to piston crank mechanism. The main feature of this mechanism is that the piston is dwell in the TDC by the $30^{\circ} \mathrm{CA}$ and thus allows combustion of the fuel in constant volume. Authors presented the process of designing an analytical cam. The starting point for the calculation was the displacement of piston graph and the limits of acceleration and velocity values obtained. The results of strength calculations of the cam with regard to fatigue problems are also presented. Calculations were carried out in order to reduce the weight of the cam. For this purpose, the Finite Element Method was used.

Key words: cam engine, analitical cam, dynamic analysis, fatigue strength, finite element method

\section{Introduction}

The most common form of conversion of piston displacement to rotary motion is the crank-piston mechanism (CrPM).

This mechanism has several advantages: compact design, low number of components, low complexity, high durability in heavy load. The schematic diagram of the crank-piston mechanism used in internal combustion engines (ICE) is shown in Fig. 1.

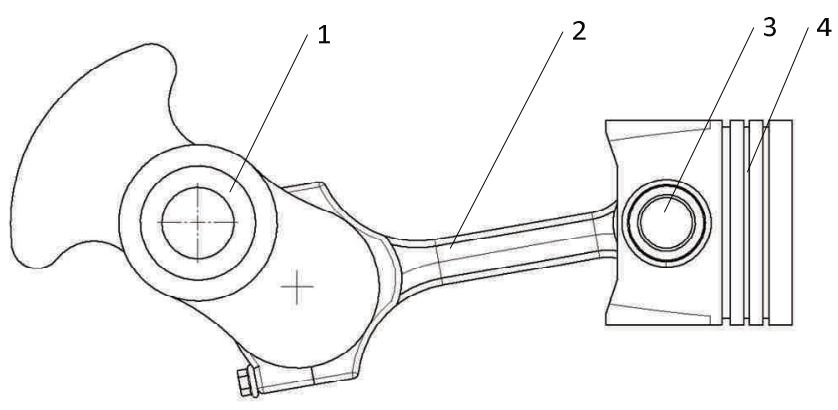

Fig. 1. Crank-piston mechanism: 1 - crankshaft, 2 - connecting rod, 3 - piston pin, 4 - piston

In the discussed mechanism the displacement of the piston $\mathrm{x}(\varphi)$ realizes the motion according to the relation (1):

$$
\mathrm{x}(\varphi)=\mathrm{R}\left[(1-\cos \varphi)+\frac{\lambda}{4}(1-\cos 2 \varphi)\right]
$$

were: $\mathrm{R}$ - crank radius, $\varphi$ - crank angle, 1 - connecting rod length, $\lambda=\frac{1}{\mathrm{R}}$

From the above equation it follows that the displacement is related to trigonometric relationships with the geometric parameters of the system. Because the feature of trigonometric functions ( $\sin$ and cos) is their continuity and variability, the course of the piston's movement is even and smooth. In addition, this feature also applies to derivatives of displacement: velocity and acceleration. Movement of a mechanism with such characteristics, which is advantageous for mechanical reasons, is less adapted to other phenomena occurring in ICE. This is referred inter alia to: cylinder filling, compression, exhaust, camshaft and valve cooperation, and most in relation to the combustion phe- nomena. Therfore, in the last few years, it has been noted that scientists and engineers have been pushing for changes to the existing CrPM or to change at the ideological level. One of the proposed methods is to use a cam-piston mechanism (CPM). The drawing of this mechanism is shown in the Fig. 2.

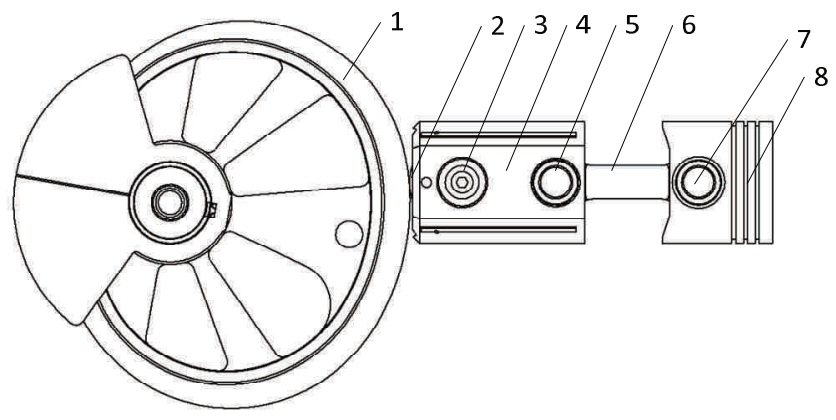

Fig. 2. Cam-piston mechanism (desctiption in the text)

In these systems, a drive cam (1) cooperates with the roller-bearing (2) mounted on a pin (3) which is associated with the piston rod (4) connected to the piston (8) by means of the connector (6) with has two more pins (5), (7). Historically one of the first uses of the CPM was Daniela's 1906 cam engine [1]. The feature of the CPM is the lack of a clear connection between the cam and the roller bearings. Therefore, an additional structural design should be used in the CPM to enable the reciprocating motion of the piston rod assembly with the cam. In ICE, this may be an element that co-operates with a recess in the lateral face of the cam or in a multi-cylinder engine, a stabilizing mechanism (for example, in the Fairchild-Caminez airplane engine [2]). From a mechanical point of view, the complexity of CPM is greater than CrPM. However, considering that the displacement of the piston is due to the outline of the cam, it can be adapted, for example, to duration of the combustion process or to the limit heat flux by the cylinder wall. Many theoretical papers have been produced in this subject, eg [3-5]. Optimizing methods are used to determine the curvature of the cam, usually by selecting the appropriate weight for the phenomena described by mathematical formulas. In addition, aspects related to the kinetics and dynamics of the 
piston movement, as well as the strength aspects, are taken into account. This subject is described in this article. However, a discussion of the outline was adopted, which was not determined only by the limitations of thermodynamic nature. It was assumed that the piston in the TDC should be stopped by a certain angle of rotation of the cam. Below is a description of the outline and the strength aspects of the adopted cam.

\section{Determining the outline of the cam}

There are many methods of determining the outline of the cam, with detailed description in the literature of the subject eg. [6-8]. The issue can be solved directly by proposing a cam profile defined eg by arcs, and then calculating the displacement, velocity, and acceleration. In addition, using the Mulitibody Dynamics method, the outline process can be automated using a feedback loop that returns specific geometric parameters of the curves (such as radii and arc lengths). By using the directly method, it may be that the search for the optimum is time consuming and often also limited.

The second way is to solve the inverse problem, ie with the assumed movement, speed and acceleration, determine the outline of the cam. In this way, the so-called Analytical Cam. If the displacement of the cam is related to the angle of rotation (the most common case) the result of the outline is obtained in polar variables. In practice, often used readymade functions representing a particular type of movement. For example, it may be harmonic or cycloidal motion. Note that harmonic or cycloidal motion describes the trigonometric relationships, and thus (as mentioned above) make the motion smooth and even. Algebraic and diagrammatic representation of these types of motion are shown in Fig. 3.

a)

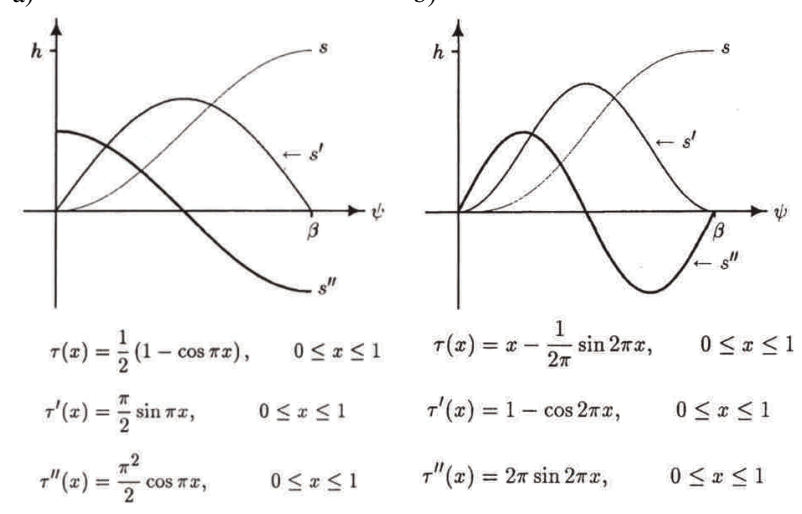

graph near the origin of the coordinate system (Fig. 2a and 2b).

However, it is not always possible to "fit" the existing and practically described types of motion to a specific application of the cam profile. One of the methods is to assume the course of the displacement and describe the mathematical relationship. In addition to the trigonometric functions, polynomial functions or spline curves can be used. For they are differentiable functions and comfortable during the search for a solution. An additional feedback method can also be used between the analyzed movements of deceplecament, speed and acceleration to conclude some compromise and optimization.

In this publication the authors present an analytical method, the motion parameters are described by polynomial functions whose base was appropriately modified trigonometric function. The starting point was the need to take into account the outline of the cam constant radius, which is in line standing piston TDC by $30^{\circ} \mathrm{CA}$. Changing the outline of the cam so that the piston's proper movement was not over $360^{\circ} \mathrm{CA}$ (as in the traditional $\mathrm{CrPM}$ ) but at $330^{\circ} \mathrm{CA}$. The additional restriction was taken into account by the need for a relatively mild increase in acceleration after dwell in TDC to avoid impact loads. For the base function of assumed harmonic motion as discussed above (see. Fig. 3a). However, in this case, the acceleration at the start point is the maximum value, which is not acceptable. As a result of a series of attempts, it was found that the displacement would be described by a grade 12 polynomial, whose course is based on the 12 characteristic points that belong to the graphs describing the motion of the cam. The general form of the polynomial shown in the following relationship:

$$
\tau(x)=a_{0}+a_{1} x+a_{2} x^{2}+\ldots+a_{n} x^{n}
$$

The solution to the problem is to find polynomial coefficients from $a_{0}$ to $a_{11}$. To this end, for each point of the characteristic equation must be placed for which the independent variable is the angle of rotation and the dependent variable the value of the displacement, velocity and acceleration. It should be added that the considered cam has a symmetrical profile, so it is justified to consider half of the cam movement. In this case it is the movement of the piston from the TDC to the BDC.

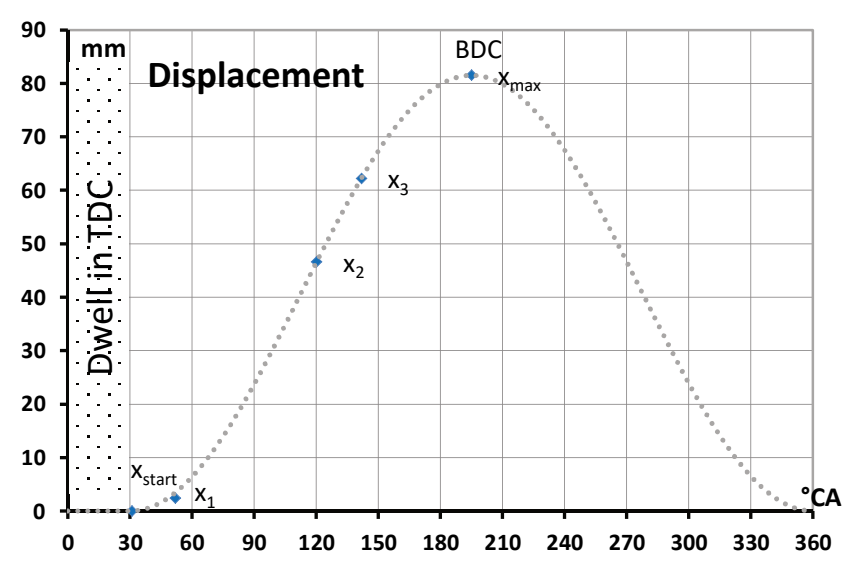

Fig. 4. Characteristic points of displacement (blue points) against the background of the base function (gray line) 
Thus, for the displacement, characteristic points were created which, in the background of the displacement described by the harmonic function, are shown in Fig. 4. Minimal point $\mathrm{x}_{\text {start }}$ in which the downward movement begins after stopping in TDC, compatible with the base function. The degree of polynomial growth between $\mathrm{x}_{\text {start }}$ and $\mathrm{x}_{1}$ depends on the number of derivatives that have the same value at this point. Point $\mathrm{x}_{1}$ does not lie on the base function. The position of the point $\mathrm{x}_{1}\left(52^{\circ} \mathrm{CA}\right)$ forces the polynomial to have an even more benign increase. The following characteristic points $\left(\mathrm{x}_{2}, \mathrm{x}_{3}, \mathrm{x}_{\max }\right)$ belong to the base function. It is easy to see that in order for a polynomial to accurately reproduce the base function, more points are needed. Limiting their numbers makes it possible to select characteristic points not only related to the displacement, but related to speed and acceleration. You can go to the description of the speed points by discussing the $\mathrm{x}_{\max }$ point. At this point, the polynomial describing the displacement should take the maximum value. The first derivative achieves zero value so the speed should be zero. As a consequence, the next characteristic point is the $\mathrm{x}_{\max }$ point but applied to the velocity graph (Fig. 5). Since at this point the speed is zero, and it relates to a derivative of the displacement.

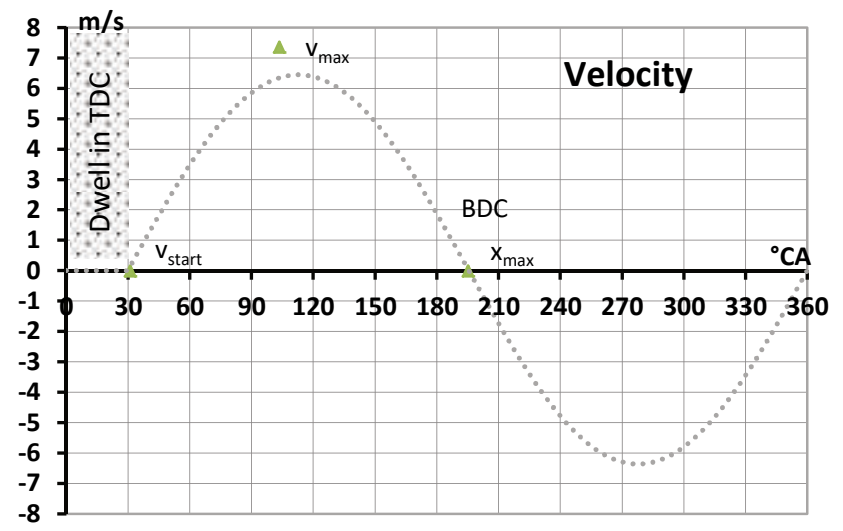

Fig. 5. Characteristic points of velocity (green points) against the background of the base function (gray line)

Since at this point the speed is zero, and it relates to a derivative of the displacement of the additional support of a polynomial (the following equation in the system of equations 12 ). For $103^{\circ}$ it was determined that the speed should have a maximum value. Before solving the system of equations, it was not defined how much it should be. So a similar procedure was used for the one described above. Namely zero of the first derivative of the speed (acceleration) provides for obtaining the maximum speed $\mathrm{v}_{\max }$ at a point. Above, it was mentioned that at the starting point (for the $30^{\circ} \mathrm{CA}$ ) is required for continuity for the second derivative included. The next characteristic point must take the same value as $\mathrm{x}_{\text {start }}$, and thus: $\mathrm{x}_{\text {start }}=\mathrm{v}_{\text {start }}=0$. The same value at this point has acceleration $a_{\text {start }}=0$ (Fig. 6). Acceptance of zero acceleration at this point deviates significantly from base acceleration, which at this point assumes a maximum value $\left(1000 \mathrm{~m} / \mathrm{s}^{2}\right)$. It is primarily for this reason could not be accepted harmonic motion to determine the outline of the cam. In point $a_{\text {start }}$ can be taken further by the value of derivatives 0 to further slow down the growth of the function. In this case it was considered that the increase was satisfactory. At the next characteristic point $\mathrm{a}_{\max }$ was given a specific angle parameter and the acceleration value. After performing a series of simulations solution and the constraints imposed by the other maximum value of the acceleration was set at $1330 \mathrm{~m} / \mathrm{s}^{2}$ at the $56^{\circ} \mathrm{CA}$. In amin also applied to a specific solution regarding the acceleration, which is at $-1350 \mathrm{~m} / \mathrm{s}^{2}$. The smoothed run in the area of the point is obtained by the condition of the continuity of the third derivative (jerk).

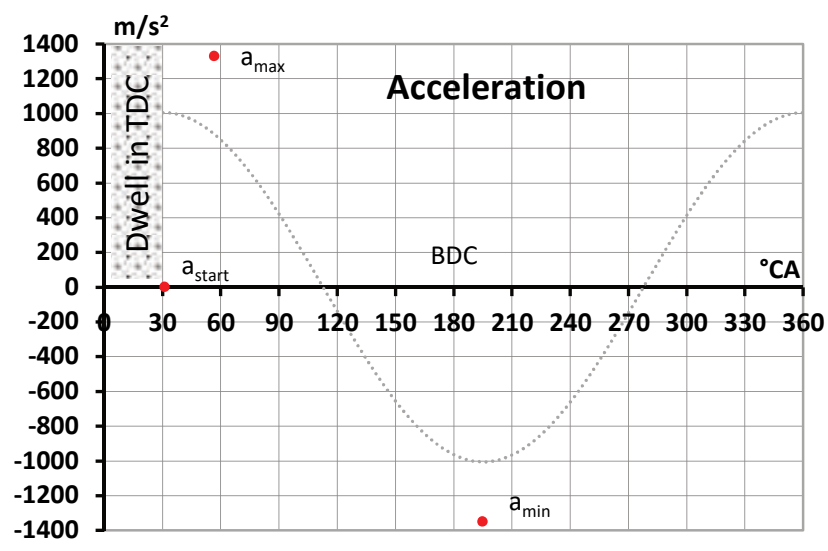

Fig. 6. Characteristic points of acceleration (red points) against the background of the base function (gray line)

The smoothed run in the area of the point is obtained by the condition of the continuity of the third derivative (jerk). This condition also causes the transition to a turning point between delay and acceleration to be smooth. The created system of equations was solved by the inverse matrix. In this method, the system of equations is written in matrix form: $\mathbf{M a}=\mathbf{B}$, where matrix $\mathbf{M}$ is a matrix of polynomial coefficients, matrix a of unknown matrix and $\mathbf{B}$ is solutions matrix. Considering the previously defined characteristic points and their values, as well as the fact that the output function is a polynomial $\tau(\mathrm{x})$ of degree 12 , a derivative of a polynomial of degree 11 , a second derivative of a polynomial of degree 10 and respectively the third derivative of a polynomial of degree 9 , the system of equations written in the matrix form is as follows:

$$
\left[\begin{array}{c}
\tau\left(x_{\text {pocz }}\right) \\
\tau^{\prime}\left(x_{\text {pocz }}\right) \\
\tau^{\prime \prime}\left(x_{\text {pocz }}\right) \\
\tau\left(x_{1}\right) \\
\tau\left(x_{2}\right) \\
\tau\left(x_{3}\right) \\
\tau\left(x_{\max }\right) \\
\tau^{\prime}\left(x_{\max }\right) \\
\tau^{\prime \prime}\left(x_{\max }\right) \\
\tau^{\prime \prime \prime}\left(x_{\max }\right) \\
\tau^{\prime \prime}\left(v_{\max }\right) \\
\tau^{\prime \prime \prime}\left(a_{\max }\right)
\end{array}\right] *\left[\begin{array}{c}
a_{0} \\
a_{1} \\
a_{2} \\
a_{3} \\
a_{4} \\
a_{5} \\
a_{6} \\
a_{7} \\
a_{8} \\
a_{9} \\
a_{10} \\
a_{11}
\end{array}\right]=\left[\begin{array}{c}
f\left(x_{\text {pocz }}\right) \\
f^{\prime}\left(x_{\text {pocz }}\right) \\
f^{\prime \prime}\left(x_{\text {pocz }}\right) \\
f\left(x_{1}\right) \\
f\left(x_{2}\right) \\
f\left(x_{3}\right) \\
f\left(x_{\max }\right) \\
f^{\prime}\left(x_{\max }\right) \\
f^{\prime \prime}\left(x_{\max }\right) \\
f^{\prime \prime \prime}\left(x_{\max }\right) \\
f^{\prime \prime}\left(v_{\max }\right) \\
f^{\prime \prime \prime}\left(a_{\max }\right)
\end{array}\right]
$$

According to the adopted methodology, the solution of the equations is a matrix solution (designated polynomial 
coefficients describing the displacement of the piston) determined from the relationship: $\mathbf{a}=\mathbf{M}^{-} \mathbf{1} \mathbf{B}$. The calculated and base polynomials are shown in the graphical interpretation in Fig. 7.

The described methodology for determining the individual points of interest results in a smoother increase in displacement after the piston is stationary at TDC $\left(30-90^{\circ}\right)$. Then, practically until the maximum stroke is reached, the course of the polynomial approximates the course of the base function. However, there are some exceptions in the range of the obtained inflection points.

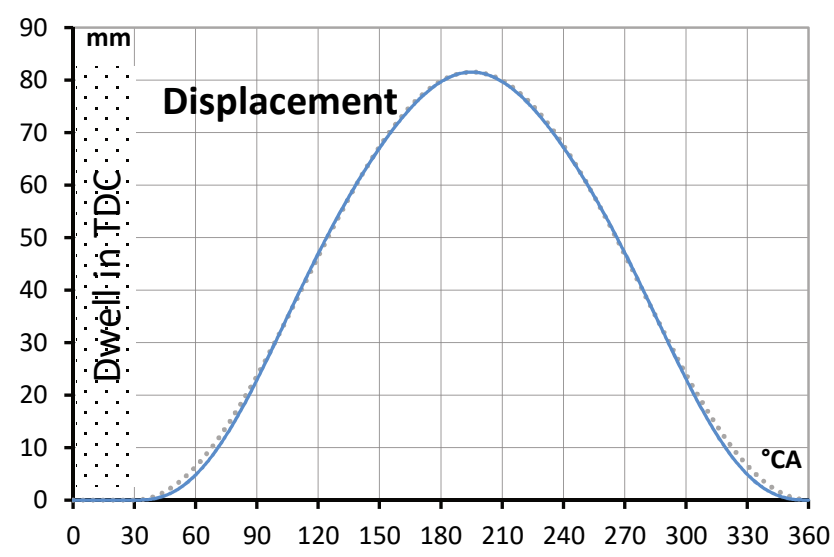

Fig. 7. Calculated displeacement (red line) against the background of the base function (gray line)

More complete description can be obtained by observing the course of the other two quantities: velocity and acceleration. It is preferable to observe them on a single graph (Fig. 8).

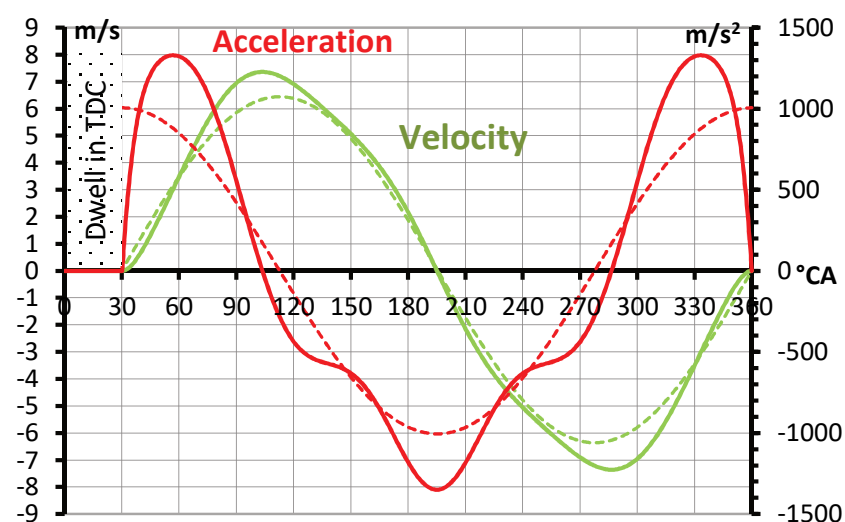

Fig. 8. Calculated velocity and acceleration against the background of the base functions

The even movement of the piston in the vicinity of the TDC, which is mentioned many times, causes the velocity and acceleration of the piston to flow smoothly. The calculated maximum speed at point $\mathrm{v}_{\max }$ (for $103^{\circ} \mathrm{CA}$ ) is 7.35 $\mathrm{m} / \mathrm{s}$. Naturally, this point acceleration value is zero. The TDC also imposed specific conditions for the acceleration achieved and its smoothing (the condition of a continuous third derivative). Adopting this condition explains both the inflection points on the displacement graph and the velocity and acceleration line between $103^{\circ}$ and $195^{\circ}$. After obtain- ing a zero value at $103^{\circ}$, the acceleration must be adjusted to the minimum value of $-1350 \mathrm{~m} / \mathrm{s}^{2}$. It can be estimated that acceleration could be developed more smoothly, without jerk, but at the expense of obtaining a minimum value at a higher level (absolute value). Changing the zero point of acceleration (maximum speed) eg $120^{\circ}$ would also change acceleration and no jerk also. Computational simulations also indicate that in such a situation the maximum velocity will increase. Since the polynomial of degree 12 , taking the point $x_{1}$ for angle values instead of $52^{\circ}$ eg. $57^{\circ}$ can lead to much greater deviation from the base function of all three sizes, and consequently to the adoption of a more complex outline of cam. The final outline of the cam, derived from the displacement of the piston, after changing the polarized to cartesian coordinates, is shown in Fig. 9.

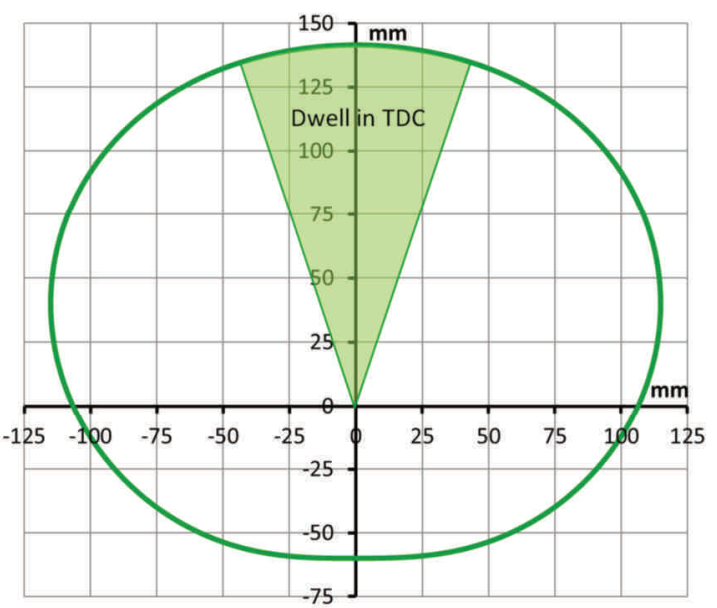

Fig. 9. Final result of cam's outline

\section{Strength test of the cam}

The engine drive system was subject to strength tests using the finite element method. The tests were performed to assess the structural strength of the object. The question of the strength rating of the track surface layer was consciously omitted. This aspect was the subject of other analyzes, which wasn't presented here.

Three-dimensional parabolic, quadrilateral finite elements (Fig. 10) were used to construct the computational model. The strength of the spline connection was determined using simple mathematical relationships, known in the specialistics literature [9]. At the location of the grooves was left a hole with internal diameter of the spline. Then translation ability of all the nodes lying on such cylinder was blocked.

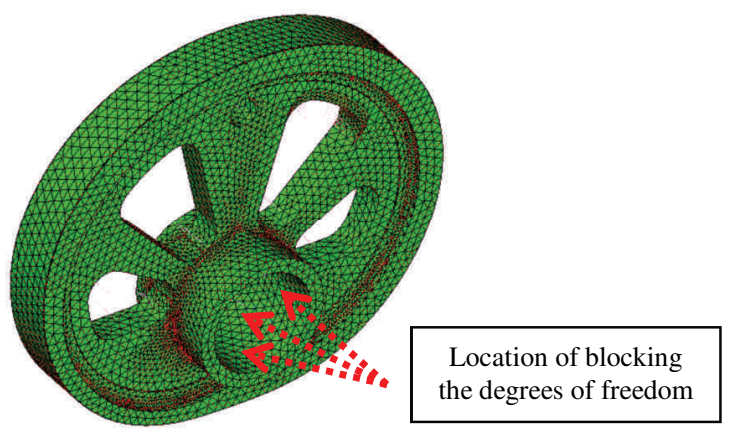

Fig. 10. Computational model of the cam 


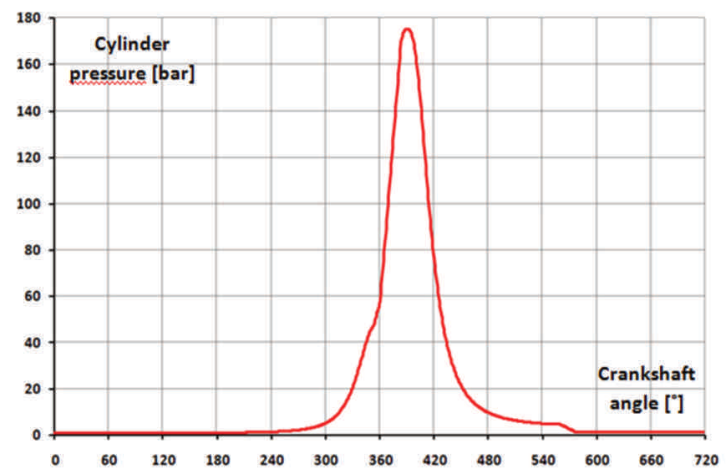

Fig. 11. Pressure inside the cylinder as a function of the angle of rotation of the crankshaft

The loads were modeled using concentrated forces. These forces was applied at right angles to the track. Variability of loads resulting from combustion process was considered (Fig 11.). The maximum load value is $73 \mathrm{kN}$ for a crankshaft angle of $390^{\circ}$, measured from TDC. Strength studies was started since then. Subsequently the stresses were evaluated with a step of $3^{\circ}$ up to 420 (Fig. 12).

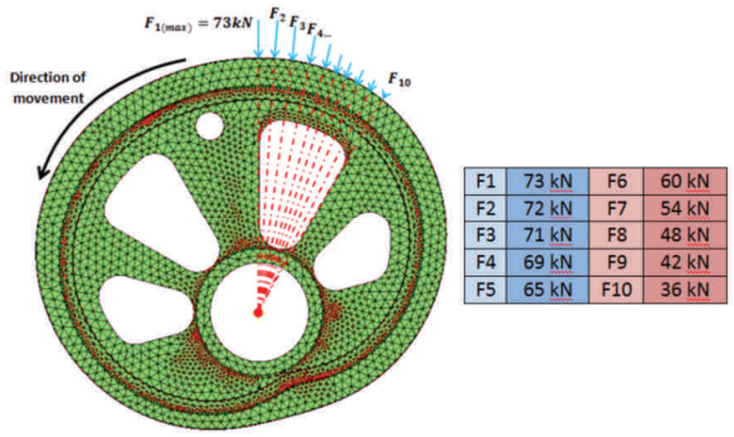

Fig. 12. Loads which was implemented in strength analysis of the cam

The forces acting on the system induce a complex state of stress. Wherein the greatest stresses occur in the cam section above the opening. This area is largely subject to bending. The selection of the geometric parameters of the crosssection, including the determination of its height, was the most important objective of the analysis. The computational model was prepared so that multivariate analysis could be carried out for different heights. Hence, four volumes were separated, which were filled with finite elements according to the diagram depicted on Fig 13.

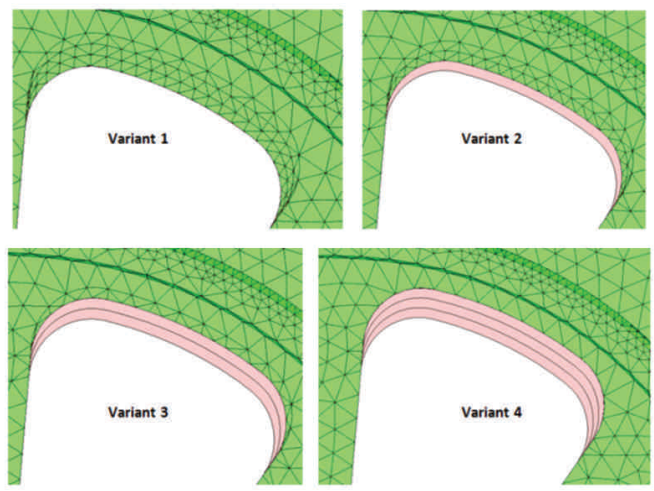

Fig. 13. Four variants of the hole shape
Below the results of calculations for two variants (1 and 4) was presented. Images depict maps of reduced tension according to Huber-Mises-Henck's hypothesis (Fig. 14 and Fig. 15). H-M-H stresses for variant 1 reach a maximum of $240 \mathrm{MPa}$, while for variant 4 about $300 \mathrm{MPa}$. These stresses accumulate in the rounding of the hole and can cause fatigue effects in this area. Due to the dominance of bending, it is necessary to select the material which has a endurance limit for bending more than $300 \mathrm{MPa}$. This feature has 18 HGT steel. Thus the carrying capacity of the cam does not deviate from the classical crank-rod system for similar mass. The system is capable of carrying heavy loads.

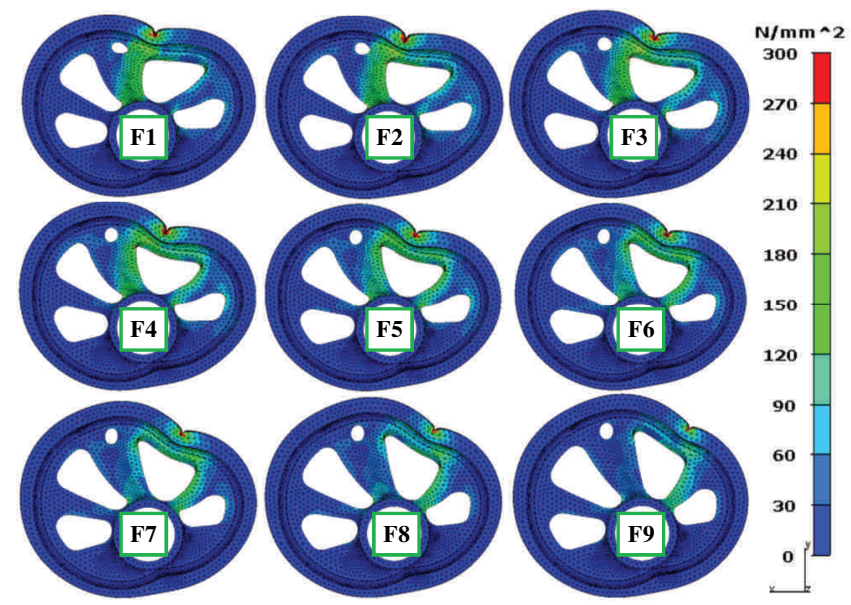

Fig. 14. Stress map H-M-H for variant 1 - displacement of nodes at a scale of 400: 1

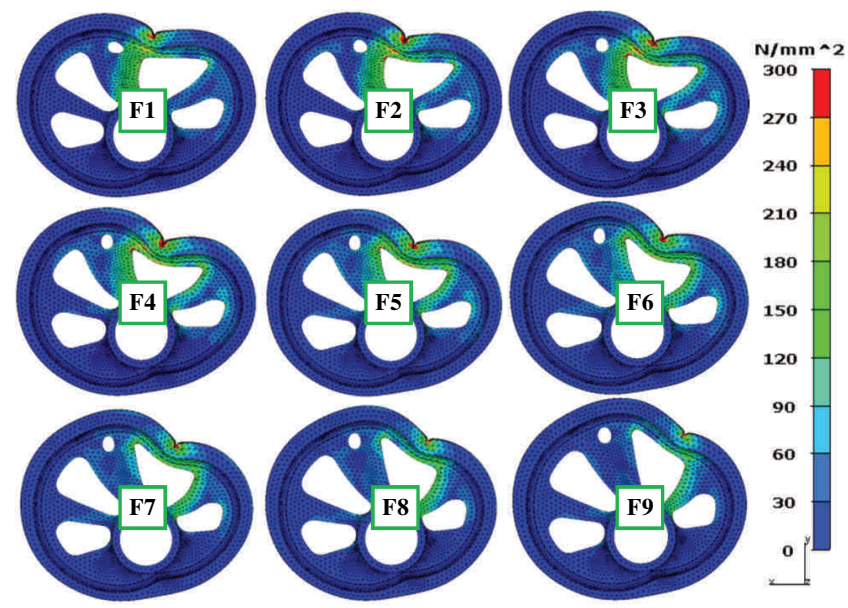

Fig. 15. Stress map H-M-H for variant 4 - displacement of nodes at a scale of 400: 1

Reduction of weight and moment of inertia is important in mechanism with high rotational speed. The presented strength test method allowed to define the shape of the cam (especially the defining of shape of openings). Removing the material will have a positive effect on the dynamics of movement. This treatment will reduce the weight of the counterweight, which is very desirable. The amplitude of the vibration of the whole engine is reduced. In addition, the applied strength verification technique, based on the finite element method, allowed to determine the geometric parameters of the cam which do not affect the ability to 
define the intended path of the piston movement. In particular, the thickness and width of the contact plane, the height of the lining and the radius of the roundings were determined. Strength indicators of material were also determined.

It is noted that the method applied is simplified (among others due to imperfections in the shape mapping, deviations from the actual material indicators, numerical method of solving the task). Hence, these are preliminary calculations. Prior to placing an object on the market, further analyzes and tests should be done.

\section{Summary}

The article describes the CPM as an alternative to the $\mathrm{CrPM}$. The main feature of the designed CPM is the piston dwell in the TDC for $30^{\circ} \mathrm{CA}$. This feature introduces significant modifications to the combustion process in the ICE. It enables: greater use of energy potential of low calorie fuels, impact on combustion kinetics and reduction of toxic compounds in exhaust gases. Performing this type of mechanism requires the use of appropriate design methods: an analytical design of the cam outline with kinematic limitations and the use of advanced FEM stress-strain estimation methods to select its geometric parameters for overpiston pressure distribution. When designing outline of the cam, the focus was on achieving a smooth and even motion of the piston. For this purpose, an analytical method with the implementation of polynomial functions was used. The smooth motion of the piston was obtained by limiting the value of the motion parameters as well as the continuity of the derivatives at the characteristic points. The beneficial effect of this method was most evident at the time of acceleration from a standstill cam at TDC. The applied calculation method allow multiple kinematic equations to solve and make interaction between the various parameters in order to have a smoother course of the modeled size. The strength tests made it possible to reduce the mass of the cam, hence it was possible to select low weight masses and low moment of inertia. The procedure for selecting the counterweight, flywheel and endurance of cam's surface layer will be presented in future publications of the authors.

\section{Nomenclature}

ICE internal combustion engine

CrPM crank-piston mechanism

CPM cam-piston mechanism
TDC top dead centre

BDC bottom dead centre

CA crank angle

\section{Bibliography}

[1] www.douglasself.com (Online).

[2] www.google.com.gt/patents/US1711260 (Online).

[3] GE, Y., CHEN, L., SUN, F. Optimal paths of piston motion of irreversible diesel cycle for minimum entropy generation. Thermal Science. 2011, 15(4), 975-993.

[4] BURZLER, K.H. Optimal piston paths for diesel engines. Thermodynamics of Energy Conversion and Transport. 2000,7 .

\footnotetext{
Tomasz Borowczyk, DEng - Faculyy of Machines and Transport at Poznań University of Technology.

e-mail: TomaszBorowczyk@wp.pl
}

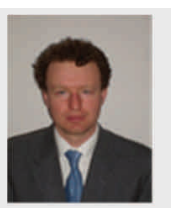

[5] BADESCU, V. Optimal piston motion for maximum net output work of Daniel cam. Energy Conversion and Management. 2015, 101, 181-202.

[6] American Can Company, Cam Design, Emmerson, 1961.

[7] ANGELES, J., LOPEZ-CAJUN, C.S. Optimization of cam mechanism. Springer Science, Business Media Dordrecht, 1991.

[8] NORTON, R.L. Cam design. Design of machinery. McGraw-Hill, 1999, 8.

[9] DIETRICH, M. Podstawy budowy maszyn. PWN. Warszawa 1988. 